families. As part of $\mathrm{BiB}$, children completed three tasks of WM when they were aged 7-10 years. Ethnicity data were collected through classroom records $(n=14,076)$, and socioeconomic data were linked from $\mathrm{BiB}$ baseline (at birth) $(n=4916)$. Linear regression was used for all analyses. WM was analysed by age to provide an interpretation of the magnitude of the effect between socioeconomic and ethnic groups. We next analysed WM by (1) a latent-class measure of socioeconomic position at birth (with least deprived as baseline) and (2) nine different ethnic groups (White British - ethnic majority - as baseline). Finally, WM scores were presented by ethnic-specific groups of socioeconomic position for the ethnic majority and largest ethnic minority group (White British and Pakistani).

Results The difference between the least and most deprived socioeconomic groups was equivalent to at least a 1-year age difference $(B=-6.02$ [95\% CI -7.51 to -4.54$])$. In comparison to White British children, Gypsy/Irish Traveller children had the lowest WM scores $(B=-9.58[-11.93$ to -7.23$]$ ) (equivalent to a two-year age difference). Most other ethnic minority children scored higher than White British children for $\geq 1 \operatorname{task}(\mathrm{s})$, for example, Pakistani children had higher scores on the forwards digit recall task $(B=3.12$ [2.53 to 3.71$]$ ) (equivalent to a 9-month age difference). Finally, there was a social gradient in WM for White British children, but not for Pakistani children.

Conclusion Given the strong associations between WM and learning ability and the potential consequences for lifelong trajectories of health and wellbeing, these large socioeconomic and ethnic group differences in children's WM are concerning. This study also found socioeconomic disadvantage was more detrimental for WM among ethnic majority children than for ethnic minority children; this may suggest that the negative effects of disadvantage are buffered by other factors for ethnic minorities, such as social support and own ethnic density.

\section{OP78 COMMUNITY SEVERANCE AND HEALTH - A NOVEL APPROACH TO MEASURING COMMUNITY SEVERANCE AND EXAMINING ITS IMPACT ON THE HEALTH OF ADULTS IN GREAT BRITAIN}

${ }^{1}$ Melissa Higgsmith*, 'Jemima Stockton, ${ }^{2}$ Paulo Anciaes, 'Shaun Scholes, 'Jennifer $S$ Mindell. 'Epidemiology and Public Health, University College London, London, UK; ${ }^{2}$ Centre for Transport Studies, University College London, London, UK

\subsection{6/jech-2021-SSMabstracts.78}

Background Aspects of community severance (the separation of people from goods, services, and each other by busy roads or other transport infrastructure) have been linked to poor health and wellbeing, but few studies have examined the overall relationship between community severance and health. We created a novel index for community severance and estimated its association with the self-rated health of adults in Great Britain.

Methods Data were collected from a nationally-representative online panel survey of 4,111 participants, February-July 2016. To construct an index, polychoric factor analysis (suitable for ordinal variables), was conducted on four survey items related to the perceived impact of roads on ability to walk locally: 1) traffic volume, 2) traffic speed, 3) availability of crossing points, and 4) adequate crossing time. Community severance index scores were negatively skewed, and were thus categorised into four groups (lowest 40\%, second, third and highest). Logistic regression was used to examine the association of community severance with self-rated health ('good' (very good/good) vs. 'poor' (fair/bad/very bad)), adjusting for potential confounders (age, income, employment status). We also examined effect modification of the severance and selfrated health association by environment type (urban/not urban) and the presence of a car in the household.

Results Polychoric factor analysis confirmed that it was appropriate to combine the four survey items into a single index (Cronbach's Alpha $=0.86$; Keiser-Meyer-Olkin measure of sampling adequacy $=0.76$, all factor loadings $>0.74$ ). After controlling for confounding factors, being in the highest community severance index group was associated with higher odds of reporting poor self-rated health (Odds Ratio: 1.79, 95\% Confidence Interval: 1.48-2.17) compared with the lowest scoring group. There was a dose-response gradient, with those in the second and third highest groups having increased odds of reporting poor self-rated health, though of lower magnitude ((OR 1.21, 1.01-1.45) and (OR 1.41, 1.16-1.71) respectively).

Conclusion We found an inverse association between community severance index and self-rated health. This suggests that to improve health, local governments and road authorities should take steps to reduce community severance through traffic reduction and calming, pedestrian prioritisation, and the installation of well-designed crossing points.

\section{Friday 17 September}

\section{Nutritional EPI \& Data, $13.00-15.30$}

\section{OP79 ENERGY AND NUTRIENT TRENDS OF MENU ITEMS SERVED BY LARGE UK CHAIN RESTAURANTS, 2018- 2020}

Yuru Huang*, Jean Adams, Thomas Burgoine. MRC Epidemiology Unit, University of Cambridge, Cambridge, UK

\subsection{6/jech-2021-SSMabstracts.79}

Background Increased consumption of out-of-home food is one important contributor to rising obesity rates. Currently, little is known about trends in the nutritional content of restaurant foods in the UK. The objective of this study was to evaluate longitudinal trends in energy and nutrient (i.e., saturated fat, sugar, and salt) content of menu items served by large UK chain restaurants.

Methods Data on energy and nutrient content of menu items served by large UK restaurant chains- defined by either number of outlets or turnover - that provided nutritional information on their websites were collected annually (20182020). A total of 23,911 items from 29 large UK chain restaurants were included in the analysis. We used linear mixed models to estimate per-item energy and nutrient changes, in all items and common items (i.e., items that were available in all three years, $\mathrm{N}=2,433$ ) over time. We also explored if the trends varied across different types of restaurants (e.g. cafés, full-service restaurants) and food categories (e.g. beverages, pizza).

Results The sugar content of menu items served by large UK chain restaurants declined $(0.43 \mathrm{~g} /$ year, $95 \% \mathrm{CI}=-0.66$, $-0.21, \mathrm{p}<0.001)$ from 2018 to 2020 . This reduction was 